\title{
Stability of Top- and Bottom-Gate Amorphous Polymer Field-Effect Transistors
}

\author{
S. Georgakopoulos*, D. Sparrowe**, F. Meyer**, M. Shkunov* \\ *Nano-Electronics Centre, Advanced Technology Institute, University of Surrey, Guildford GU2 7XH, UK \\ **Merck Chemicals Ltd., Chilworth Technical Centre, University Parkway, Southampton SO16 7QD, UK
}

Performance and stability between the top- and bottom-gate field-effect transistor configurations are investigated in dual-gate transistor structures consisting of the same insulator and gate materials. The transistors behave similarly for both gate modes with ON/OFF ratio in excess of $10^{5}$, subthreshold swing of $0.5-1 \mathrm{~V} /$ decade, and mobility of $0.03-0.04$ $\mathrm{cm}^{2} / \mathrm{Vs}$, retained over several months, with fabrication, storage, and characterization, performed in ambient conditions.

Polymer field-effect transistors (PFETs) are promising candidates for the fabrication of low-cost electronic circuits and pixel drivers, due to the potential of depositing all device elements from solution, into layered thin-film structures and onto flexible substrates ${ }^{1}$. The position of the gate and co-planar source/drain electrodes with respect to the active layer (above or below), define one of four different geometries. Studies on source/drain electrode position (bottomcontact, top contact) have revealed differences in terms of contact resistance, subthreshold swing, mobility and stability ${ }^{2,3,4}$.

Dual-gate (DG) PFETs, consisting of both a top-gate (TG) and a bottom-gate (BG) in the same device structure, have been studied in recent years ${ }^{5,6,7}$, as the configuration offers increased control of certain device parameters such as the turn-on voltage. However, the differences in PFET performance and stability between TG and BG have not been examined extensively. Also, reported DG work is mainly based on asymmetric insulator-gate structures, where passivated $\mathrm{Si} / \mathrm{SiO}_{2}$ is a convenient choice for the bottom gate/insulator, whereas polymer insulator/metal is typically used for the top gate/insulator.

In the present work we have developed symmetric DG structures (Fig. 1a) with thermally evaporated Au TG/BG (40 $\mathrm{nm}$ thick) and spin-cast Cytop ${ }^{\mathrm{TM}}$ (Asahi Glass) top-/bottom-insulator (900/850 nm thick respectively). Au source and drain electrodes (40 nm thick) were produced by thermal evaporation, photolithography, and iodine-based $\left(\mathrm{I}: \mathrm{KI}: \mathrm{H}_{2} \mathrm{O}\right)$ etching, to form interdigitated finger structures with channel length $\mathrm{L}=10 \mu \mathrm{m}$ and width $\mathrm{W}=1 \mathrm{~cm}$. The performance and stability between TG- and BG-PFETs is compared over a period of 143 days in air by examining variations across 3 transistors which are operated exclusively in single gate bias, whilst the second gate is left to float.

The fluoropolymer Cytop ${ }^{\mathrm{TM}}$ exhibits good properties for a PFET insulator, including low dielectric permittivity $\left(\varepsilon_{\mathrm{r}}=\right.$ 2.1-2.2) and thus minimal energetic disorder at the semiconductor-insulator interface ${ }^{8}$, and hydrophobic surface, safeguarding against moisture-related instabilities (mobility degradation and turn-on voltage shifts ${ }^{9}$, and hysteresis ${ }^{10}$ ). However, the hydrophobic surface poses a problem for further deposition from solution. The issue can be overcome if the surface tension is first modified by exposure to plasma species ${ }^{11}$. In the present work the surface was exposed to $50 \mathrm{~W}$ oxygen plasma for $30 \mathrm{~s}$, resulting in no observable roughness increase $(0.5-2 \mu \mathrm{m}$ area scale), as examined by tappingmode AFM. The treatment allowed for spin-coating of a $150 \mathrm{~nm}$ thick active layer of poly(tetraoctylindenofluorine-alttriarylamine) (PIF8-TA1, Fig. 1b).

The trade-off for this surface treatment is a positive shift of the turn-on voltage for $\mathrm{BG}\left(\mathrm{V}_{\mathrm{ON}}=4 \mathrm{~V}\right)$ (Fig. 2), which is undesirable as the PFET is ON under zero gate bias. TG exhibits slightly negative turn-on voltage $\left(\mathrm{V}_{\mathrm{ON}}=-2 \mathrm{~V}\right)$ arising from the fluorine terminated Cytop ${ }^{\mathrm{TM}}$ fluoropolymer surface. The turn-on voltage for both gate modes remains stable over the period of characterization. The turn-on voltage stability of this insulator/semiconductor combination for extensive biasing periods has been examined elsewhere and found to be insignificant ${ }^{12}$.

Semiconductor PIF8-TA1 was prepared by Suzuki polymerization from dibromoindenofluorine and diboronate ester triarylamine monomers and dissolved in toluene $(10 \mathrm{mg} / \mathrm{ml})$. The class of indenofluorine-triarylamine co-polymers exhibit amorphous morphology ${ }^{13}$, allowing a more direct comparison between the top and bottom semiconductorinsulator interfaces, without concern for structure variations. PFETs utilizing this material have been shown to exhibit consistent and reproducible charge carrier mobilities across a range of deposition techniques ${ }^{12}$. Additionally, this class of polymers has exhibited good stability, suggested to originate from the high ionization potential (IP) (5.4 - $5.5 \mathrm{eV}$ by photoelectron spectroscopy in air) ${ }^{13}$.

In the present work, the HOMO level for PIF8-TA1 was found to lay at $5.45 \pm 0.1 \mathrm{eV}$ by cyclic voltammetry on a Princeton Applied Research VersaSTAT 4. To match the HOMO level with the Au Fermi level and eliminate injection barriers, source/drain electrodes were treated with pentafluorobenzenethiol in ethanol ( $0.5 \mathrm{mMol}, 10$ minute immersion), resulting in an effective workfunction increase of up to $1.1 \mathrm{eV}$ as compared to untreated Au surface. Measurements were taken with a McAllister Kelvin Probe KP6500 in air.

PFETs were fabricated, stored, and characterized in ambient conditions. Transistor measurements were taken with a Keithley 4200-SCS semiconductor parameter analyzer. For output scans the drain bias was swept in $0.5 \mathrm{~V}$ steps and no injection non-linearities or voltage drops were observed throughout the characterization period. For transfer scans the gate bias was swept from 10 to $-30 \mathrm{~V}$ in $0.5 \mathrm{~V}$ steps, and drain bias stepped from $-2 \mathrm{~V}$ (linear regime) to $-30 \mathrm{~V}$ (saturation regime). Turn-on voltages were extracted from linear regime semi-logarithmic transfer scans at $I_{D}=1 p A$. Turn-on 
voltages for the saturation regime were observed to be positive shifted by $0.5 \mathrm{~V}$ as compared to linear regime. Forward and reverse transfer scans showed no measurable hysteresis for either gate mode throughout the characterization period.

Fig. 3 shows the typical linear-regime transfer scans of one of the devices operated either in TG or BG mode. Each plot contains 11 scans taken over 143 days. For TG, no degradation trend can be observed and the subthreshold swing is $0.5 \mathrm{~V} /$ decade. For BG, the subthreshold swing is closer to $1 \mathrm{~V} /$ decade and the slope of the curve somewhat degrades over time (Fig. 3 inset). The subthreshold swing is linked to the trap density at the interface ${ }^{14}$, and oxygen-induced acceptor-states have been shown to increase its value in pentacene FETs ${ }^{15}$. The subthreshold swing has also been found to be affected by the channel geometry ${ }^{16}$, thus it could be inherently different for the TG and BG configurations.

Field-effect mobilities were extracted with standard MOSFET equations (1) and (2) for linear and saturation regime respectively from transfer scans ${ }^{17}$. Saturation mobilities are peak values of curved profiles. Linear regime mobilities were extracted in the $\mathrm{V}_{\mathrm{G}}=20-30 \mathrm{~V}$ range.

$$
\begin{gathered}
\mu_{L I N}=\frac{d I_{D}}{d V_{G}} \frac{L}{C_{i} W V_{D}} \\
\mu_{S A T}=\left(\frac{d \sqrt{I_{D}}}{d V_{G}}\right)^{2} \frac{L}{C_{i} W}
\end{gathered}
$$

The capacitance per unit area $\left(\mathrm{C}_{\mathrm{i}}\right)$ was calculated from the measured insulator thickness (Tencor Alpha-step, scratch and measure trench depth). A small deviation from actual values is likely present, and in combination with access resistance for TG, could have resulted in slightly different effective mobilities for TG and BG.

The saturation-regime mobility for TG well stabilizes at $0.03 \mathrm{~cm}^{2} / \mathrm{Vs}$ after $1-2$ months, while for BG lays into the range of $0.03-0.04 \mathrm{~cm}^{2} / \mathrm{Vs}$ (Fig. $4 \mathrm{~b}$ ). The obtained values are in close agreement with reported values of $0.026 \pm 0.009^{12}$ $\mathrm{cm}^{2} / \mathrm{Vs}$ and $0.04^{13} \mathrm{~cm}^{2} / \mathrm{Vs}$ of semiconductors of similar structure. The degradation rate is not significant and appears similar for both TG and BG. In the linear regime (Fig. 4a) we observe a noticeably higher mobility degradation rate for BG, at least initially. The degradation in the linear regime for BG (linear mobility and slope of transfer curve) but not in the saturation regime suggests instability at the source/drain-semiconductor contact.

In summary, similar performance is observed for both gate modes with the exception for BG of a positive turn-on voltage shift, caused by the oxygen plasma treatment, and a contact degradation issue, the origin of which cannot be ascertained from the available data. The PFETs presented here exhibit highly stable performance in air, as opposed to thiophene-based semiconductors ${ }^{18}$, and even passivated pentacene FETs ${ }^{19}$.

\footnotetext{
${ }^{1}$ H. Sirringhaus, T. Kawase, R.H. Friend, T. Shimoda, M Inbasekaran, W. Wu, E.P. Woo. Science 15, 2123 (2000)

${ }^{2}$ R.A. Street, A. Salleo. Appl. Phys. Lett. 81, 2887 (2002)

${ }^{3}$ I.G. Hill. Appl. Phys. Lett. 87, 163505 (2005)

${ }^{4}$ A. Herasimovich, S. Scheinert, I. Horselmann. J. App. Phys. 102, 054509 (2007)

${ }^{5}$ F. Maddalena, M. Spijkman, J.J. Brondijk, P. Fonteijn, F. Brouwer, J. C. Hummelen, D.M. de Leeuw, P.W.M. Blom, B. De Boer. Organic Electronics 9, 839 (2008)

${ }^{6}$ M. Spijkman, E.C.P. Smits, P.W.M. Blom, D.M. de Leeuw, Y. Bon Saint Come, S. Setayesh, E. Cantatore. Appl. Phys. Lett. 92, 143304 (2008)

${ }^{7}$ K. Hizu, T. Sekitani, T. Someya. Appl. Phys. Lett. 90, 093504 (2007)

${ }^{8}$ J. Veres, S.D. Ogier, S.W. Leeming, D.C. Cupertino. Adv. Funct. Mater. 13, 199 (2003)

${ }^{9}$ H. Sirringhaus. Adv. Mater. 21, 3859 (2009)

${ }^{10}$ Y.H. Noh, S.Y. Park, S.M. Seo, H.H. Lee. Organic Electronics 7, 271 (2006)

${ }^{11}$ M. Uno, Y. Tominari, J. Takeya. Organic Electronics 9, 753 (2008)

${ }^{12}$ J. M. Verilhac, M. Benwadih, A. L. Seiler, S. Jacob, C. Bory, J. Bablet, M. Heitzman, J. Tallal, L. Barbut, P. Frere, G. Sicard, R. Gwoziecki, I. Chartier, R. Coppard, C. Serbutoviez. Organic Electronics 11, 456-462 (2010)

${ }^{13}$ W. Zhang, J. Smith, R. Hamilton, M. Heeney, J. Kirkpatrick, K. Song, S. E. Watkins, T. Antonopoulos, I. McCulloch.

J. Am. Chem. Soc 131, 10814-10815 (2009)

${ }^{14}$ W.L. Kalb, B. Batlogg. Physical Review B 81, 035327 (2010)

${ }^{15}$ D. Knipp, T. Muck, A. Benor, V. Wagner. Journal of Non-Crystalline Solids 352, 1774 (2006)

${ }^{16}$ J.M. Verilhac, M. Benwadih, S. Altazin, S. Jacob, R. Gwoziecki, R. Coppard, C. Serbutoviez. Appl. Phys. Lett. 94, 143301 (2009)

${ }^{17}$ Z. Bao, J. Locklin. Organic Field-Effect Transistors, CRC Press, $1^{\text {st }}$ ed., p.554 (2007)

${ }^{18}$ Q. Xia, M. Burkhardt, M. Halik. Organic Electronics 9, 1061 (2008)
} 
${ }^{19}$ G.W. Hyung, J. Park, J.H. Kim, J.R. Koo, Y.K. Kim. Solid State Electronics 54, 439 (2010)

Figures:

a

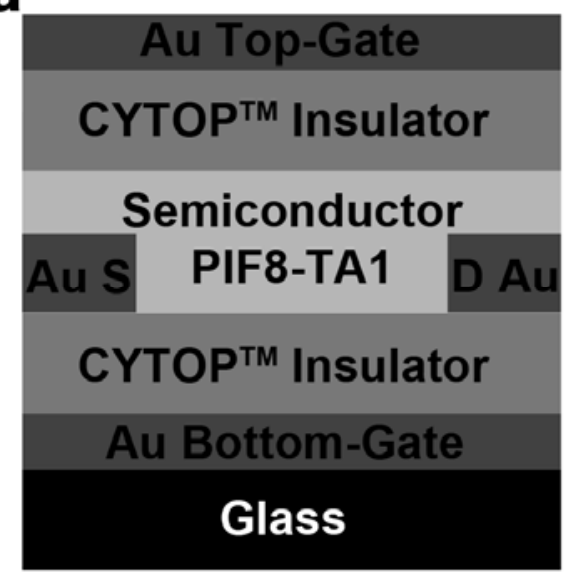

Fig. 1. $\quad$ a) Layer structure of DG PFET , co-polymer structure.

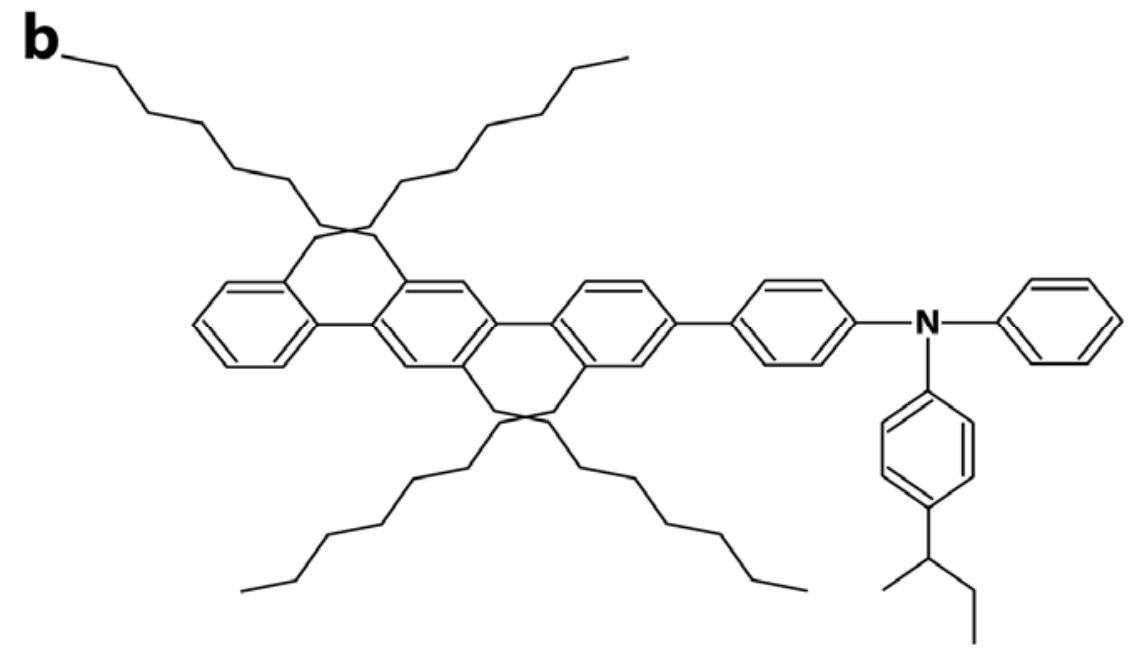

b) poly(tetraoctylindenofluorine-alt-triarylamine) (PIF8-TA1) amorphous $\pi$-conjugated

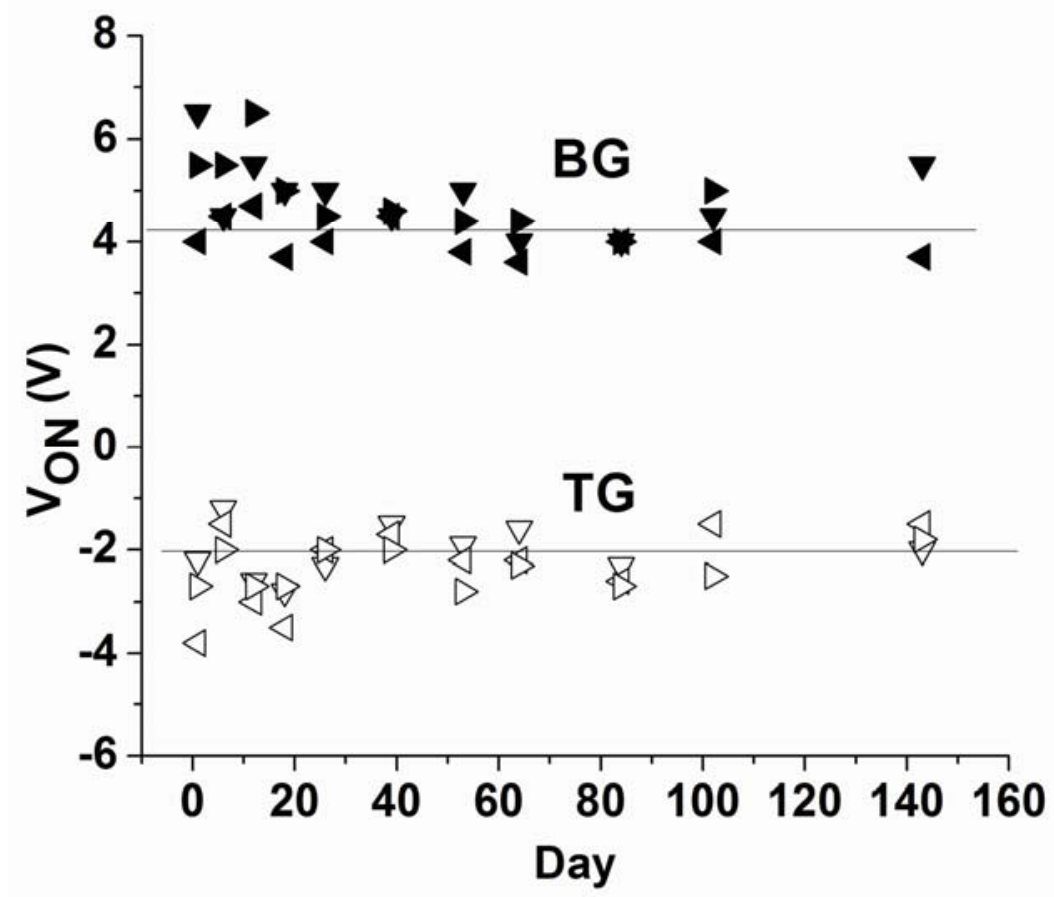

Fig. 2. Progression of turn-on voltage of 3 DG PFETs represented by down-/left-/right-facing triangles, for BG (fill) and TG (no fill), over 143 days. Up/down/left-facing triangles represent different devices. 

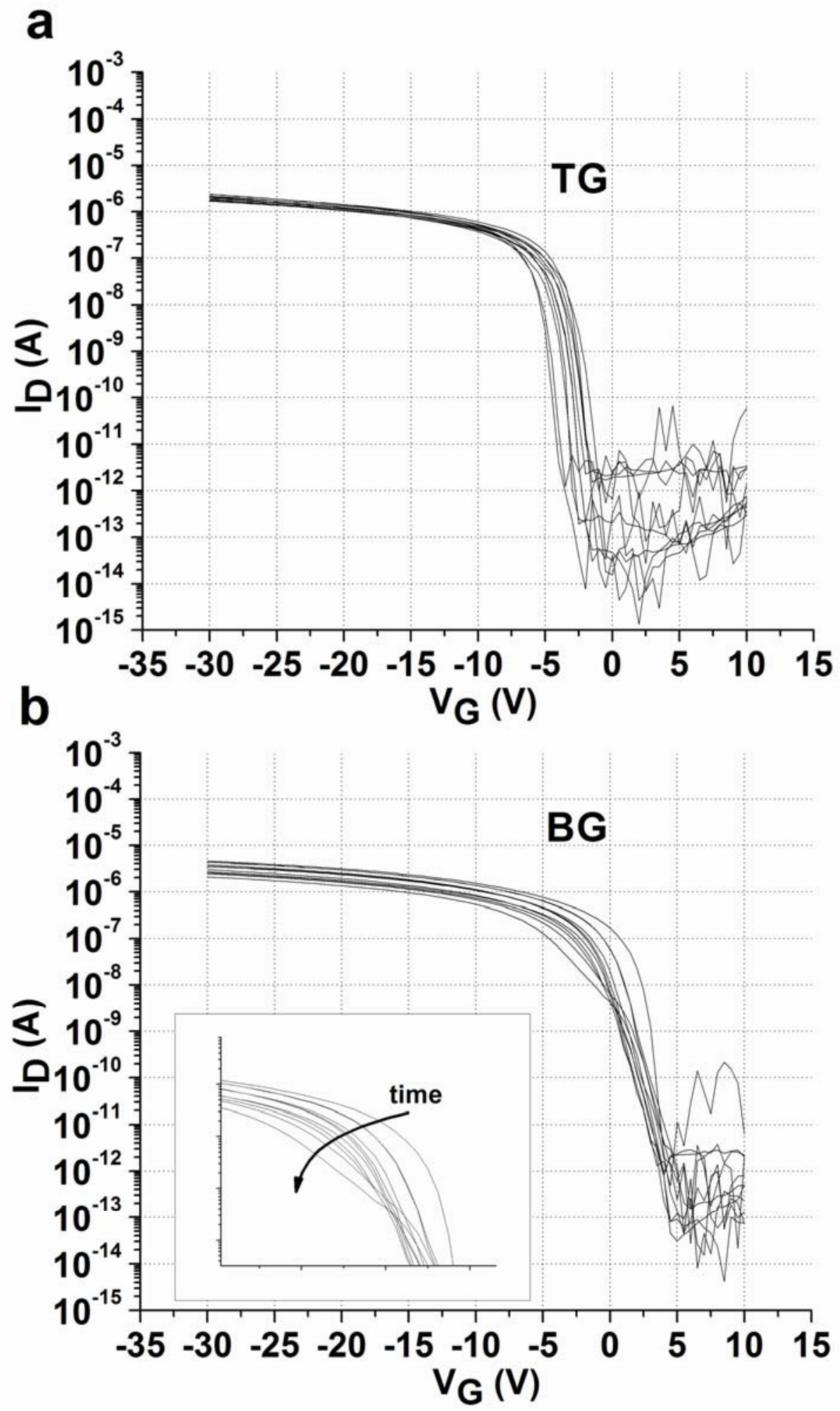

Fig. 3. Forward transfer scans of DG PFET in linear regime $\left(V_{D, \text { LIN }}=-2 \mathrm{~V}\right)$ for a) $\mathrm{TG}$ and $\left.\mathrm{b}\right) \mathrm{BG}$. Scans taken at different points in time, ranging from 1 to 143 days after fabrication. There is no clear trend over time for TG. BG inset shows slope degradation over time. 


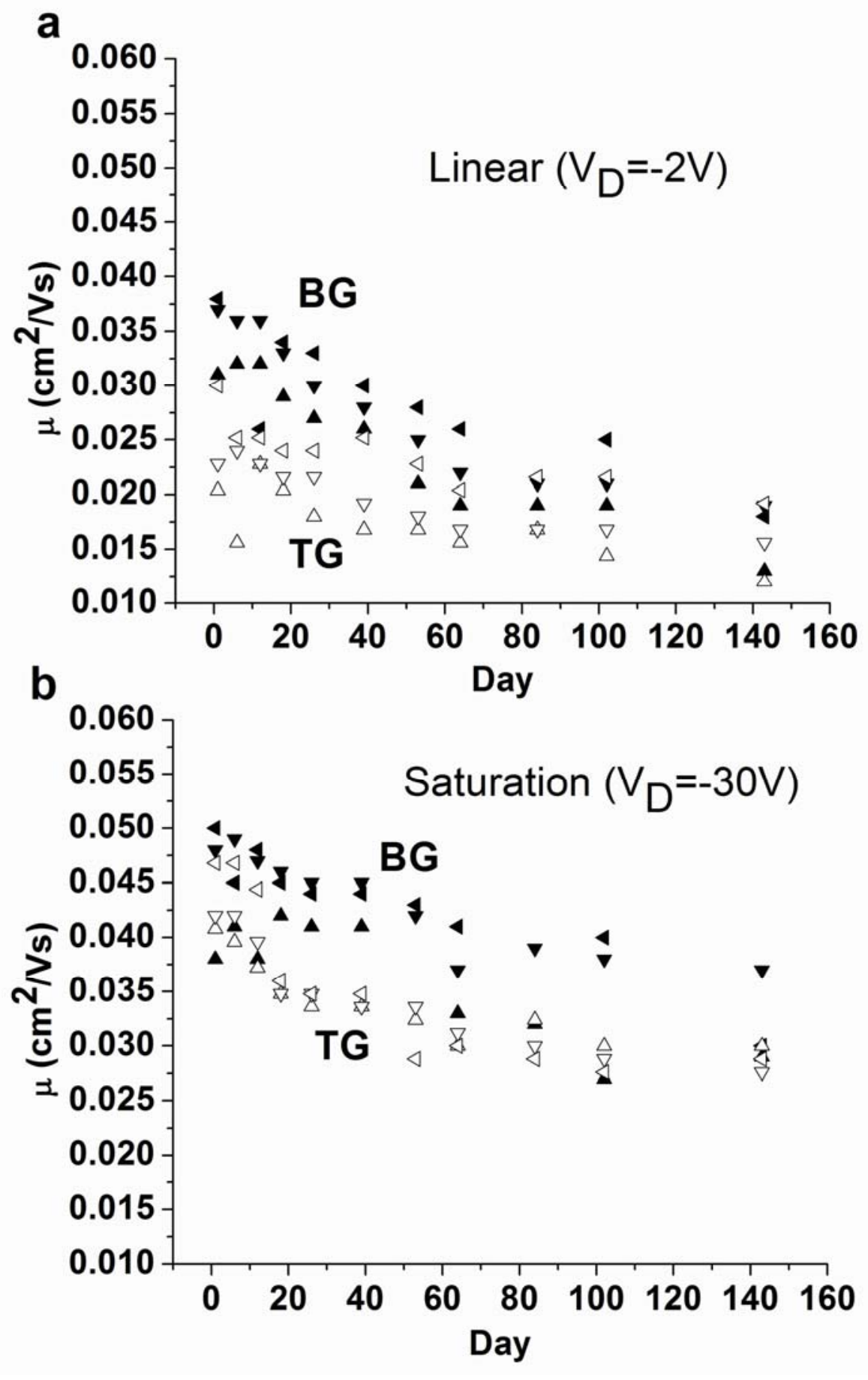

Fig. 4. Progression of a) linear $\left(V_{D}=-2 \mathrm{~V}\right)$ and $\quad$ b) saturation $\left(V_{D}=-30 \mathrm{~V}\right)$ mobilities over 143 days, for $B G$ (fill) and $T G$ (no fill). Up/down/leftfacing triangles represent different devices. 